$\begin{gathered}\text { EPiC Series in Built Environment } \\ \text { Volume 2, 2021, Pages 1-9 }\end{gathered}$
ASC 2021. 57th Annual Associated Schools
of Construction International Conference

\title{
Influence of a Back-Support Exoskeleton on Physical Demands of Rebar Work
}

\author{
Nihar Gonsalves, Omobolanle Ogunseiju, and \\ Abiola Akanmu Ph.D. \\ Virginia Polytechnic Institute and State University, \\ Blacksburg, Virginia
}

\author{
Chukwuma Nnaji, Ph.D. \\ The University of Alabama, Tuscaloosa, \\ Alabama
}

Low back injuries are a major concern in the construction industry. Rebar workers develop lower back injuries as a result of prolonged awkward positions and repetitive motions. Exoskeletons can be an effective intervention for preventing back injuries. However, little is known regarding the potential impacts of their use on physical demands of construction work. This study presents an evaluation of the effects of a commercially available exoskeleton during simulated rebar work. Assessment of the exoskeleton was based on muscle activity and range of motion of the back during placing and tying subtasks of the rebar work. With the exoskeleton, muscle activity reduced in the erector spinae group by 10-30\% during placing subtask. Latissimus dorsi muscle group showed a reduced muscle activity of $7-31 \%$ during the placing subtask but increased by $1-14 \%$ during tying subtasks. The back range of motion reduced by $17.15 \%$ during placing subtask and increased by $23.41 \%$ during the tying subtask. The reduction in muscle activations and range of motion during placing subtask agrees with the intended design of Laevo. However, the increased muscle activities and range of motion are indicative of increased discomfort and fatigue. These results could be helpful in redesigning exoskeletons to significantly reduce injury risk in construction.

Key Words: Low-back disorder, Passive exoskeleton, Rebar Work, Laevo, Muscle activation

\section{Introduction}

Musculoskeletal disorders (MSDs) are a major concern in the construction industry. Approximately $33 \%$ of all occupational injuries and illnesses requiring days away from work in the United States are attributed to work-related musculoskeletal disorders (WMSDs) (W. Umer, H. Li, G. P. Y. Szeto, \& A. Y. Wong, 2017a). In 2017, the rate of WMSDs in the construction industry was about 9\% higher than the rate of 28.6 MSD per 10,000 full-time employment (FTE) for all industries (BLS, 2020), with rebar workers being one of the construction trades with the highest rate (36.6 MSD per 10,000 FTE). Rebar workers position and secure steel bars or mesh in concrete forms to reinforce concrete. They perform this task mostly in non-neutral or awkward postures, imposing significant strain on the back. Prolonged 
and repetitive assumption of these postures results in back injuries which have significant implications on workforce health, and project productivity and cost (Chen, Qiu, \& Ahn, 2017). Ample evidence also indicates that severe back injuries result in permanent disability and premature exits from the workforce (Antwi-Afari, Li, Yu, \& Kong, 2018; Koopman, Kingma, Faber, de Looze, \& van Dieën, 2019).

Several interventions have been explored for reducing WMSDs. For example, workforce training (Akanmu, Olayiwola, Ogunseiju, \& McFeeters, 2020) and mechanical assistive devices (Albers \& Estill, 2007). There is also increasing interest in the use of wearable robots (such as back-support exoskeletons) to reduce the risk of musculoskeletal disorders. Back-support exoskeletons are designed to reduce physical demands on the back, by providing assistive moments about the hip or lower spine to support the muscles (Zhang \& Huang, 2018). Studies have shown that the deployment of backsupport exoskeletons could have intended benefits such as reducing muscle activity in the back (Bosch, van Eck, Knitel, \& de Looze, 2016; Koopman et al., 2019). Bosch et al. (2016) reported a reduction of $21-31 \%$ in erector spinae muscle activity during static holding and simulated repetitive picking and placing tasks using Laevo exoskeleton. Another study conducted on cyclic lifting and lowering tasks also reported a reduction of 12-15\% in erector spinae muscle activity (Huysamen et al., 2018). Backsupport exoskeletons could also trigger unintended consequences such as discomfort in the body parts (Kim, Madinei, Alemi, Srinivasan, \& Nussbaum, 2020; Kim et al., 2018). However, results from these studies may not be directly applicable to construction work as the tasks do not adequately capture the repetitive and physically demanding nature of construction work. Furthermore, the study participants were restricted to a specific postures during the experiment which are not representative of construction tasks. Thus, little is known of the contribution of back-support exoskeletons towards reducing the physical demands imposed by construction tasks such as rebar tasks.

\section{Research Objective}

This paper presents an ergonomic assessment of a commercially available back-support exoskeleton aimed at supporting the back during rebar work to reduce the risk of WMSDs. The objectives of the study were to assess the effect of the back-support exoskeleton on back muscle activity and range of motion.

\section{Methodology}

\section{Participants}

A convenience sample size of 10 participants completed the study and signed the informed consent form approved by the Institutional Review Board of Virginia Tech. All the participants reported having no current or prior musculoskeletal injuries. Participants were volunteer male students of Virginia Tech with the following demographic statistics in mean and standard deviation: age $=23 \mathrm{yrs} \pm 1.99$, weight $=155.70 \mathrm{lbs} . \pm 22.51$ and height $=173.40 \mathrm{~cm} \pm 4.97)$.

\section{Equipment \& Instrumentation}

\section{Exoskeleton}

This study adopted Laevo V2.56 (www.laevo-exoskeletons.com) (see figure 1), a passive back-support exoskeleton designed to reduce the load on the lower back during forward bending and repetitive tasks. Major components of Laevo include chest pad, leg pad, torso, smart joint, and hip padding. When users 
assume a bending position, the exoskeleton applies a counterforce in the opposite direction via the chest pad. This reduces the stress on the lower back and transfers the absorbed load to other body parts through the leg pads. The exoskeleton also includes three torso fittings which can be adjusted to suit the user's physique using adjustment belts.

\section{Surface electromyography}

Muscle activities were measured using Somaxis Cricket surface electromyography (EMG) sensor. The muscle activation data was collected at a sample rate of $500 \mathrm{~Hz}$ using a Chirp iOS application. The EMG communicated with the Chirp application using Bluetooth Low Energy. Four EMG sensors were placed over two groups of back muscles (i.e., the left and right latissimus dorsi and erector spinae) (see figure 2). Rebar workers perform their work in stooping and twisting positions thereby inducing significant stress in the back muscle. Erector spinae muscle group is one of the major muscles activated during the rebar work given the forward bending nature of the work (Bosch et al., 2016; Huysamen et al., 2018; W. Umer, H. Li, G. P. Y. Szeto, \& A. Y. L. Wong, 2017b). Furthermore, to identify the muscle activation due to lateral movement of trunk, latissimus dorsi muscle group were also been measured in this study (Picchiotti, Weston, Knapik, Dufour, \& Marras, 2019; Umer et al., 2017b; Weston, Alizadeh, Knapik, Wang, \& Marras, 2018).

\section{Inertial Measurement Unit}

Trunk flexion was measured using Yost labs Inertial Momentum Unit (IMU) (see figure 3). The IMU measured the flexion (i.e., range of motion) of the trunk while performing the rebar task with and without the exoskeleton. The IMU was attached to the trunk of all the participants using an elastic belt.

\section{Experimental Task Description}

Rebar task involving repetitive placing and tying (subtasks) of four prefabricated gates were simulated as representative of a typical construction task. Each gate (see figure 4) is composed of \#11 rebars placed at 2" on center (both ways). Participants were required to place the prefabricated gates on the floor and tie six joints with pre-cut rebar ties using a plier. To represent the repetitive nature of rebar task, the placing and tying of each gate represented a cycle. The participants completed four cycles with the exoskeleton (Laevo) and without the exoskeleton.

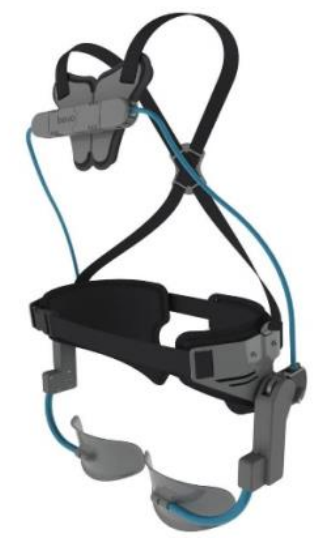

Figure 1. Laevo (V2.56) Exoskeleton

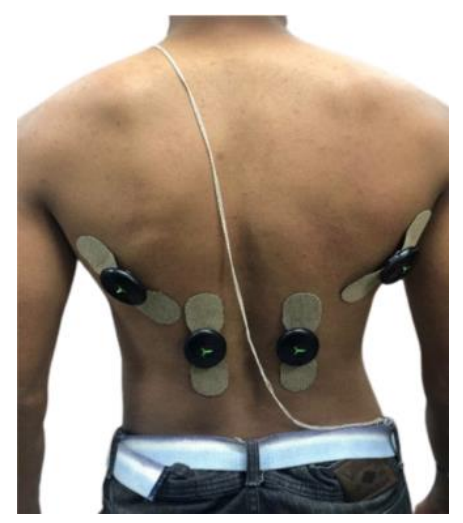

Figure 2. EMG sensors placement 


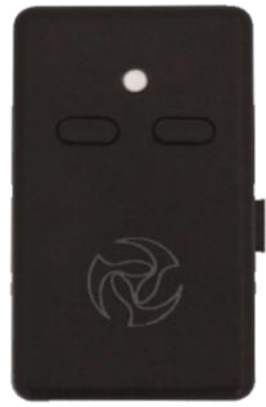

Figure 3. Yost Labs Data Logger

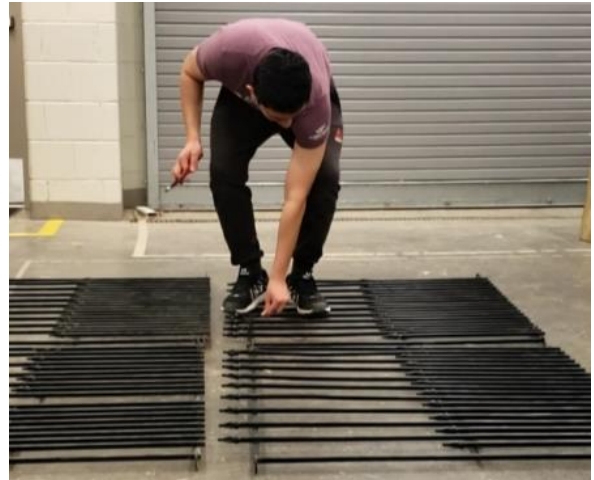

Figure 4. Simulated rebar task

\section{Procedure}

Participants were introduced to the workings of the exoskeleton and allowed to don and doff the exoskeleton without assistance. After participants were comfortable with the exoskeleton, the rebar tasks were introduced to them. To ensure accuracy and familiarity of the tasks, participants were allowed 15 to $30 \mathrm{mins}$ to complete several trials of the tasks. Thereafter, the EMG sensors were bilaterally positioned in direct contact with two muscle groups (latissimus dorsi and erector spinae) of participants' backs. Afterward, the IMU sensor was placed on the participant's back, and the placements of the EMG and IMU sensors were checked. Before commencement of the rebar task, participants were required to perform three- 10 secs Maximum Voluntary Contractions at $30^{\circ}, 45^{\circ}$, and $90^{\circ}$ each separated by one-minute rests. Thereafter, the participants performed four cycles of the rebar tasks without exoskeleton. After the task, they were allowed 15 mins rest to avoid fatigue before repeating the tasks with the use of the exoskeleton. During the experimental task, the participants were video-recorded. The recording served as ground truth for the data analysis.

\section{Data analysis}

EMG data were analyzed in two stages (pre-processing and statistical analysis). In the first stage of analysis, the raw data was band-pass filtered at 20-500 Hz. Root mean square values were then calculated using a $100 \mathrm{~ms}$ sliding window. Subsequently, normalized values were calculated using Maximum voluntary contraction (MVCs) similar to the works of Mirka (1991). These were used to compute the peak ( 90 percentile), median (50 percentile), and static (10 percentile) values to understand the effect of the exoskeleton during the static, median, and peak muscle activations. All data processing was conducted using MATLAB 2020Ra and MS Office Excel 2020.

The statistical analysis for the study was performed using $\mathrm{R}$ studio (Version 1.2.5042). Three-way repeated measure Analysis of Variance (ANOVA) was used to analyze the range of motion and muscle activations. For the EMG data, the independent variable in this experimental study includes the exoskeleton conditions (i.e., exoskeleton and no exoskeleton) in which the participant is performing the rebar task, muscle activity level (i.e., static, median, and peak), and the sub-tasks (i.e., placing and tying), and the dependent variable was the normalized EMG data. For the range of motion, the independent variables are the exoskeleton conditions, cycles, and subtasks, while the dependent variable is the range of motion. A statistically significant difference was reported in this study when P-value is less than 0.05 . 


\section{Results}

\section{Range of Motion}

A significant difference $(\mathrm{p}=0.00272)$ was observed in the range of motion (ROM) while participants performed placing and tying subtasks. Similarly, the interaction effects between exoskeleton conditions and tasks were observed $(\mathrm{p}=0.016)$. With the use of the exoskeleton, the ROM of participants during tying subtask was higher than the ROM during placing subtask when the exoskeleton was not used. Averagely, during placing subtask, the ROM was observed to be $17.15 \%$ lower while using the Laevo (i.e., Exo condition) compared to when no exoskeleton was used (i.e., Noexo condition) (see figure 5). However, the ROM during tying subtask was averagely $23.41 \%$ higher while using the exoskeleton (see figure 6).

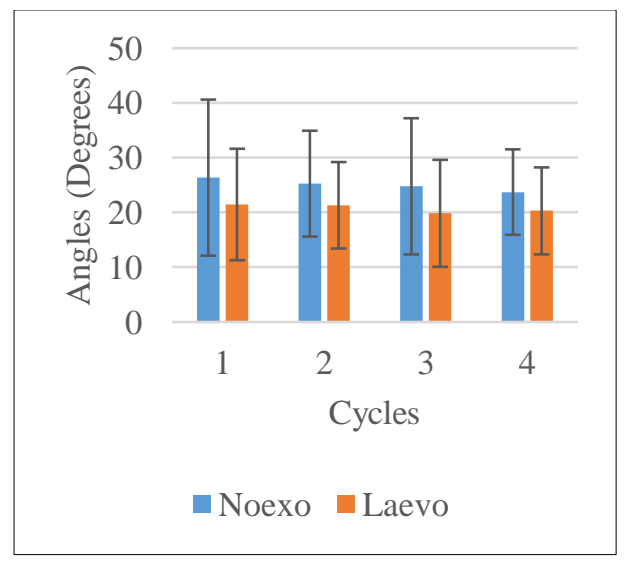

Figure 5. Range of motion - Placing subtask

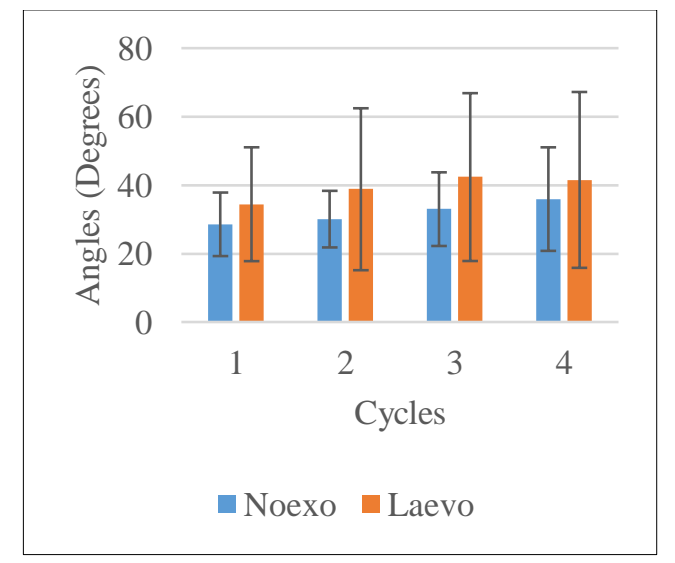

Figure 6. Range of motion - Tying subtask

\section{Left Latissimus Dorsi (LLD)}

\section{Muscle Activity}

The static, median, and peak EMG amplitude from the left latissimus dorsi for tying subtask was significantly lower compared to placing subtask. For the placing subtask, the static, median, and peak muscle activities were $7 \%, 17 \%$, and $27 \%$ respectively lower while using the Laevo exoskeleton (see figure 7) compared to Noexo condition. During the tying subtask, these values were found to be $5 \%$, $5 \%$, and $14 \%$ respectively higher while using Laevo (see figure 8) compared to Noexo condition.

\section{Right Latissimus Dorsi (RLD)}

EMG amplitude for the static, median and peak values for tying subtask was found to be significantly lower compared to the placing subtask. For the placing subtask, the static, median, and peak EMG normalized values from the right latissimus dorsi were lower while using the Laevo exoskeleton. i.e., $11 \%, 10 \%$, and $31 \%$ respectively (see figure 9) compared to Noexo condition. During the tying subtask, the mean and peak values were also found to be lower while using the exoskeleton. i.e., $6 \%$, and $8 \%$ respectively (see figure 10) compared to Noexo condition. Whereas, the static values was $1 \%$ higher when using the exoskeleton compared to the Noexo condition. 


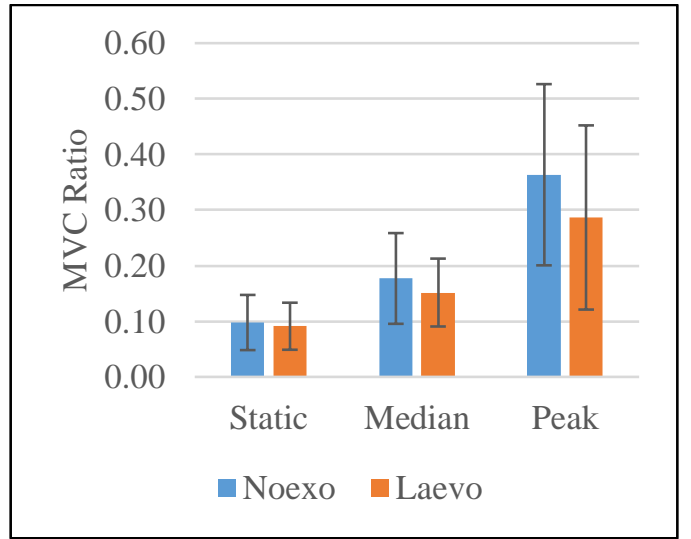

Figure 7. LLD EMG - Placing subtask

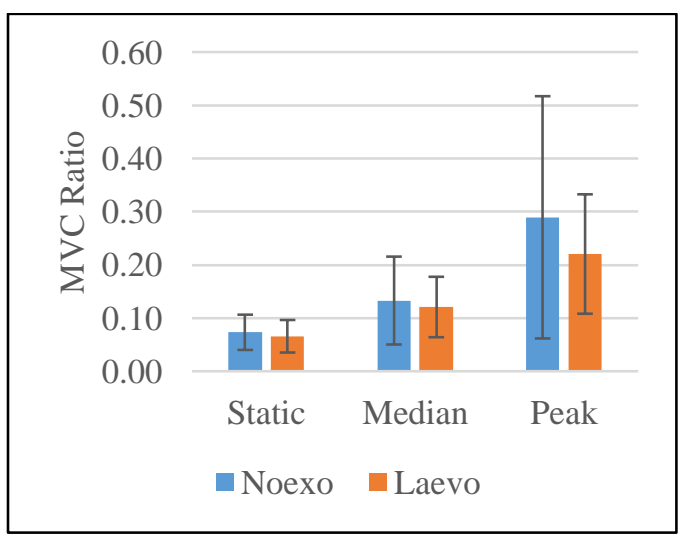

Figure 9. RLD EMG - Placing subtask

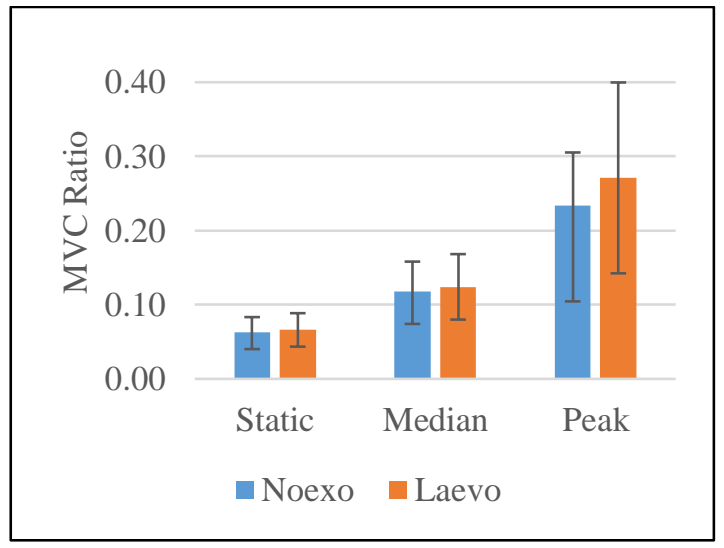

Figure 8. LLD EMG - Tying subtask

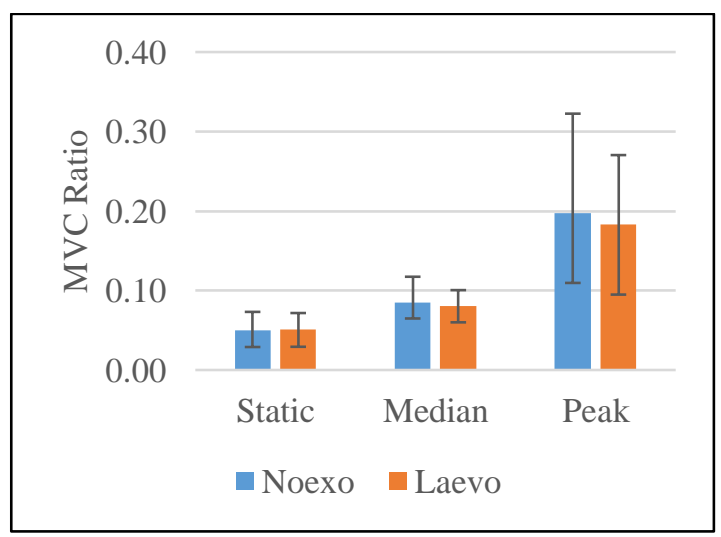

Figure 10. RLD EMG - Tying subtask

\section{Left Erector Spinae (LES)}

For tying subtask, in the left erector spinae, the static, median, and peak EMG amplitude was significantly lower compared to placing subtask. However, the impact of the exoskeleton was predominant during placing than tying subtask. The static, mean and peak values during the placing subtask were found to be $10 \%, 27 \%$ and $30 \%$ (respectively) lower when the exoskeleton was used, (see figure 11) compared to Noexo condition. For the tying subtask, the median and peak values were $4 \%$ and 3\% (respectively) lower, but there was no observed changes in the static EMG activations when the tasks were performed with the Laevo exoskeleton (see figure 12) compared to Noexo condition. 


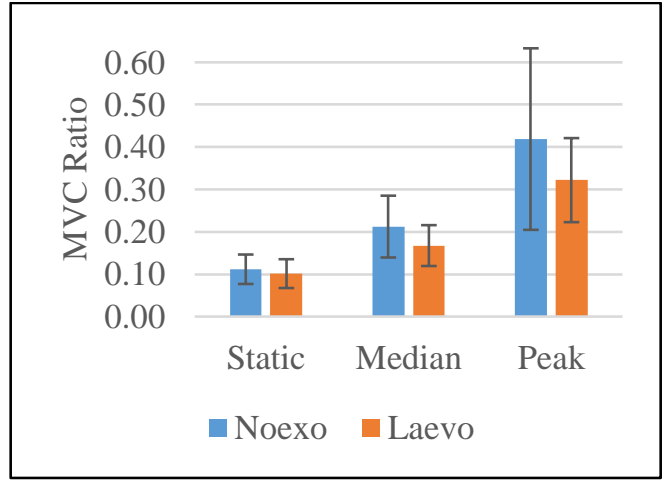

Figure 11. LES EMG - Placing subtask

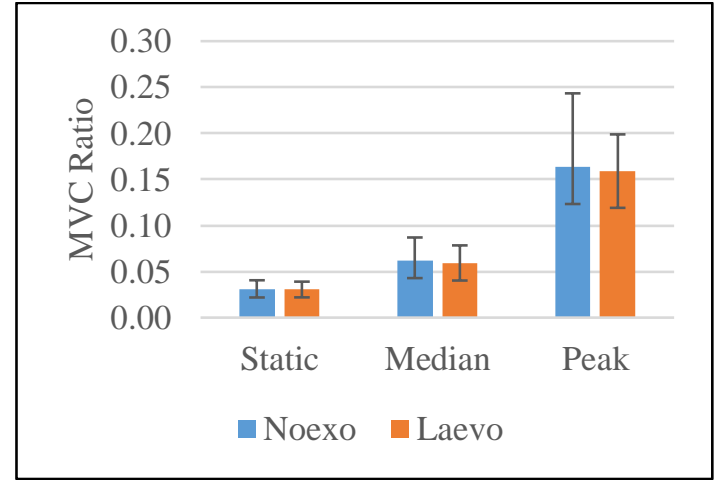

Figure 12. LES EMG - Tying subtask

\section{Right Erector Spinae (RES)}

In the right erector spinae, the static, median, and peak EMG amplitude for tying subtask were significantly lower $(\mathrm{p}=0.000138)$ compared to placing subtask. Between exoskeleton conditions and subtasks, a significant difference $(\mathrm{p}=0.0417)$ was also observed. When placing subtask was performed with the Laevo exoskeleton, the EMG amplitude was higher than when tying subtask was performed without the exoskeleton. For the placing subtask, the static, median, and peak EMG normalized values were $15 \%, 17 \%$, and $19 \%$ (respectively) lower while using the Laevo exoskeleton (see figure 13) compared to Noexo condition. During the tying subtask, the static and peak values were $6 \%$ and $4 \%$ (respectively) lower but 4\% higher for median EMG muscle activations while using the Laevo exoskeleton (see figure 14) compared to Noexo condition.

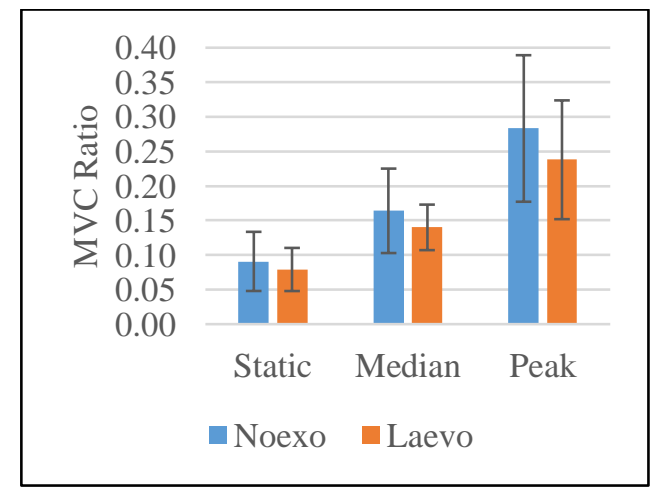

Figure 13. RES EMG - Placing subtask

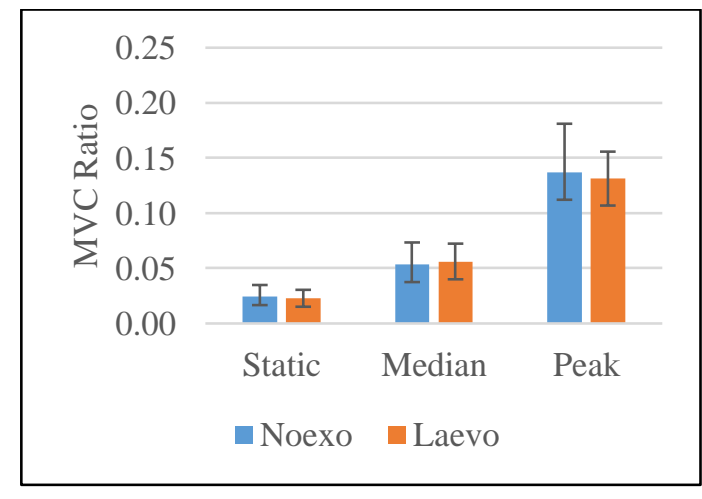

Figure 14. RES EMG - Tying subtask

\section{Discussion}

This study focused on understanding the potential impact and effectiveness of the Laevo exoskeleton for rebar construction work. The study simulated repetitive rebar tasks and employed EMG and IMU for collecting data on the muscle activities and ROM respectively. It was observed that ROM during tying subtask was substantially higher compared with placing subtask. This could also be attributed to 
the postures assumed by the participants while carrying out the task. The participants stooped to place the gates on the floor and this triggered the reduced range of motions at the back. During tying subtask, some of the participants squatted while others assumed kneeling positions, which involves increased ROM at the back.

During placing subtask, (where the participants assumed stooping or forward bending posture), the exoskeleton significantly reduced activity levels across the erector spinae and Latissimus Dorsi muscle groups. This result was consistent throughout the static, median, and peak EMG amplitudes. The findings from this study align with the intended design objective of the Laevo exoskeleton which is to relieve stress from the lower back. Another reason for the reduction in the erector spinae is the reduced ROM of the back when the exoskeleton was used. However, it was observed that during tying subtask, (where the participants assumed squatting and kneeling postures) the effect of the exoskeleton in reducing muscle actions was not consistent. Although there was a reduction in muscle activities at the Erector spinae, this was not substantial, and not consistent for the static, medium, and peak EMG amplitudes. The inconsistency in the tying subtask could be attributed to the fact that the Laevo exoskeleton is designed for forward bending task. Furthermore, there was an increase in the muscle activity levels of the left latissimus dorsi while using the Laevo exoskeleton. This could be because the exoskeleton might have restricted the movement of the participants, thereby requiring them to exert more force to complete the rebar work.

\section{Limitations}

There are some limitations in this study that will be considered in future work. Firstly, the rebar tasks were performed by the students who are novices and not experienced rebar workers. Hence, there is a possibility that experienced rebar workers might assume different postures while performing the subtasks. Thus, caution should be exercised when generalizing the results of this study to the construction industry. Secondly, this study only considered two muscle groups in the back region. Since the rebar tasks involve bending, stooping, squatting, and in some cases kneeling, muscle groups of other body parts such as the chest, thigh, leg, and abdomen could similarly be impacted. Thirdly, the long term effect of the exoskeleton on the muscle was not assessed during the study. It is important to note that the exoskeleton may have long-term effects on the muscles of the users. Thus, long-term study is required to understand the prolonged use of the back-support exoskeleton on construction workers.

\section{Conclusion and Future Work}

Construction activities often involve physically demanding and repetitive movements that result in musculoskeletal injuries. This study assessed the effectiveness of a commercially available exoskeleton for reducing back injuries during rebar work. The assessment is carried out via range of motion and muscle activations. The agreeability of reduced ROM and muscle activities during placing subtasks indicates that when used for a short time, Laevo exoskeleton supports tasks involving forward bending. Similarly, the inconsistency of the impacts of Laevo in reducing ROM and muscle activities during tying subtasks shows that awkward postures involving squatting and kneeling can trigger muscle activities in other body parts. However, since the Laevo exoskeleton works by transferring the load to the legs, there could be potential unexpected consequences in the thigh muscles. It is therefore important that manufacturers of back-support exoskeletons consider the effects of diversity in postures of construction work on the muscle activations when designing exoskeletons for the construction industry. This study contributes to existing scarce literature on the suitability of wearable robots for construction work. This study also provides data for designing virtual exoskeletons to improve future exoskeleton designs and for enhancing workforce training. 


\section{Acknowledgement}

This material is based upon work partly supported by Construction and Infrastructure Research Affiliates Program (CIRAP).

\section{References}

Akanmu, A. A., Olayiwola, J., Ogunseiju, O., \& McFeeters, D. (2020). Cyber-physical postural training system for construction workers. Automation in Construction, 117, 103272.

Albers, J., \& Estill, C. F. (2007). Simple solutions; ergonomics for construction workers.

Antwi-Afari, M. F., Li, H., Yu, Y., \& Kong, L. (2018). Wearable insole pressure system for automated detection and classification of awkward working postures in construction workers. Automation in Construction, 96, 433-441.

BLS, B. o. L. S. (2020). Nonfatal cases involving days away from work: selected characteristics (2011 forward). Retrieved from https://data.bls.gov/pdq/SurveyOutputServlet

Bosch, T., van Eck, J., Knitel, K., \& de Looze, M. (2016). The effects of a passive exoskeleton on muscle activity, discomfort and endurance time in forward bending work. Applied ergonomics, 54, 212-217.

Chen, J., Qiu, J., \& Ahn, C. (2017). Construction worker's awkward posture recognition through supervised motion tensor decomposition. Automation in Construction, 77, 67-81.

Huysamen, K., de Looze, M., Bosch, T., Ortiz, J., Toxiri, S., \& O'Sullivan, L. W. (2018). Assessment of an active industrial exoskeleton to aid dynamic lifting and lowering manual handling tasks. Applied ergonomics, 68, 125-131.

Kim, S., Madinei, S., Alemi, M. M., Srinivasan, D., \& Nussbaum, M. A. (2020). Assessing the potential for "undesired" effects of passive back-support exoskeleton use during a simulated manual assembly task: Muscle activity, posture, balance, discomfort, and usability. Applied ergonomics, 89, 103194.

Kim, S., Nussbaum, M. A., Esfahani, M. I. M., Alemi, M. M., Jia, B., \& Rashedi, E. (2018). Assessing the influence of a passive, upper extremity exoskeletal vest for tasks requiring arm elevation: Part II-“Unexpected" effects on shoulder motion, balance, and spine loading. Applied ergonomics, 70, 323-330.

Koopman, A. S., Kingma, I., Faber, G. S., de Looze, M. P., \& van Dieën, J. H. (2019). Effects of a passive exoskeleton on the mechanical loading of the low back in static holding tasks. Journal of biomechanics, 83, 97-103.

Mirka, G. A. (1991). The quantification of EMG normalization error. Ergonomics, 34(3), 343-352.

Picchiotti, M. T., Weston, E. B., Knapik, G. G., Dufour, J. S., \& Marras, W. S. (2019). Impact of two postural assist exoskeletons on biomechanical loading of the lumbar spine. Applied ergonomics, $75,1-7$.

Umer, W., Li, H., Szeto, G. P. Y., \& Wong, A. Y. (2017a). Low-cost ergonomic intervention for mitigating physical and subjective discomfort during manual rebar tying. Journal of Construction Engineering and Management, 143(10), 04017075.

Umer, W., Li, H., Szeto, G. P. Y., \& Wong, A. Y. L. (2017b). Identification of biomechanical risk factors for the development of lower-back disorders during manual rebar tying. Journal of Construction Engineering and Management, 143(1), 04016080.

Weston, E. B., Alizadeh, M., Knapik, G. G., Wang, X., \& Marras, W. S. (2018). Biomechanical evaluation of exoskeleton use on loading of the lumbar spine. Applied ergonomics, 68, 101108.

Zhang, T., \& Huang, H. (2018). A lower-back robotic exoskeleton: Industrial handling augmentation used to provide spinal support. IEEE Robotics \& Automation Magazine, 25(2), 95-106. 